\title{
Valores centrales y sociolingüística en la comunidad de habla de Almería
}

\author{
Core Values and Sociolinguistics \\ in the Almeria Speaking Community
}

\author{
Francisco García Marcos \\ Universidad de Almería, España \\ fgarcos@gmail.com
}

\begin{abstract}
The article applies in the Almeria speaking community the core value sociolinguistic methodology developed by J.J. Smolicz in Australia. The results demonstrate the absolutely nuclear role of language in that community, in all target groups. The case of Almería, very probably, shows a universal pattern of sociolinguistic behaviour. In principle, it confirms the theoretical and methodological hypothesis developed in Australia. They also highlight the relevance of interdisciplinary-based research, from which valuable descriptive results can be obtained for the disciplines involved.
\end{abstract}

Keywords: sociolinguistics, core value, attitudes, sociolinguistics stratification

\section{INTRODUCCIÓN}

Siguiendo la referencia de las investigaciones australianas de J.J. Smolicz (1979), el Círculo Andaluz de Lingüística Aplicada (CALA) ha mostrado un ya asentado interés por las investigaciones sociolingüística de valor central, o si se prefiere de core value en la terminología sajona. Smolicz adaptó a la sociolingüística criterios y métodos con un cierto peso dentro de la investigación social de aquel tiempo. En el análisis de los mercados, las proyecciones del marketing, la economía o la antropología empezaban a obtenerse resultados muy alentadores, contando con esa metodología, capaz de adentrarse en la profundidad de los sistemas de evaluación de los grupos sociales. 
La sociolingüística nunca ha mostrado reparo en adaptar metodologías de otras ciencias sociales para sus cometidos. No dejó de suceder así en su etapa fundacional en Francia o la antigua URSS, ni tampoco en sus versiones más modernas aparecidas en EE.UU. a principios de los 60 (García Marcos 1999). De manera que, en no poca medida, Smolicz seguía casi una tradición disciplinar, al adaptar y transferir esa metodología a la investigación de la interrelación entre lengua y sociedad. Hay que decir que tuvo éxito inmediato, no solo entre los especialistas, sino incluso en la sociedad en general, debido a su alto rendimiento en planificación lingüística. Los datos que proporciona el core value son más precisos y refinados que los obtenidos por los sociólogos del lenguaje norteamericanos $\mathrm{y}$, en consecuencia, permiten sustentar con mayor solidez las intervenciones que propone todo programa de planificación. Gracias a ello su aplicabilidad se incrementa de forma notoria. No es de extrañar que la repercusión de Smolicz y su propuesta teórico-metodológica fuera extraordinaria, al menos entre los especialistas más comprometidos con una visión panorámica de la sociolingüística, coinvirtiéndose a partir de ese momento en uno de sus grandes referentes internacionales.

Al menos ese era mi punto de vista cuanto traté de analizar ese modelo teórico de la lingüística, el sociolingüístico, en las puertas ya del siglo XXI (García Marcos 1999). Desde entonces, ha figurado regularmente en la nómina de preocupaciones que hemos ido abordando los sociolingüistas del CALA, lo que ha tenido su lógica e inevitable transcripción en la investigación empírica. Dirigidos desde el propio CALA, Otero y Mendy aportaron en 2014 una primera aproximación a dos comunidades tan diferentes como la provincia de Almería y la ciudad suiza de Neuchâtel, respectivamente. En 2015, por mi parte, propuse la versión definitiva de una matización teórica al planteamiento de Smolicz, a través del concepto de densidad de valores. Sin desatender la atención hacia la jerarquía organizativa de los valores, como se había realizado hasta ese momento, incidí en el grado de adhesión recibido por cada uno de ellos. Es decir, trataba de comprobar, además de la presencia/ausencia de valores, si estos se concentraban/dispersaban en todo su espectro. Por ello propuse considerar la existencia de escalas con predominio de valores periféricos (difusas), frente a otras organizadas en función de los parámetros opuestos (densas).

Los resultados de nuestras investigaciones -e incluso nuestros datos- fueron tomados por otros investigadores, proyectándolos en otras disciplinas sociales (Alías, 2016). Aunque esos trabajos no forman parte de las competencias del CALA, en todo caso no dejan de ser una caprichosa vuelta a los orígenes científicos de estas investigaciones; esto es, a ese fondo compartido por buena parte de las disciplinas sociales.

Los estudios de core value descansan en la hipótesis de que el imaginario social está integrado por un conjunto de valores. Cada grupo del espectro social selecciona unos valores y desecha otros, conformando de esa manera un patrón evaluativo de su propia colectividad. Las lenguas reflejan ese hecho, reservándole su campo semántico específico. La tercera acepción del término "valor" en el Diccionario de la Real 
Academia Español lo presenta como "alcance de la significación o importancia de una cosa, acción, palabra o frase". En términos análogos aparecería en Duden $\left(1^{\mathrm{a}}\right)$, Diccionari de la Llengua Catalana $\left(1^{\mathrm{a}}\right)$, Dictionnaire La Toupie $\left(4^{\mathrm{a}}\right)$, Oxford $\left(2^{\mathrm{a}}\right)$, Dizionario Italiano $\left(4^{\mathrm{a}}\right)$ o, entre otros, Dicionário on line de Português $\left(1^{\mathrm{a}}\right)$.

Como acaba de apuntarse, esos valores están organizados de manera jerárquica, lo que supone una gradación implícita en el imaginario de los grupos sociales.

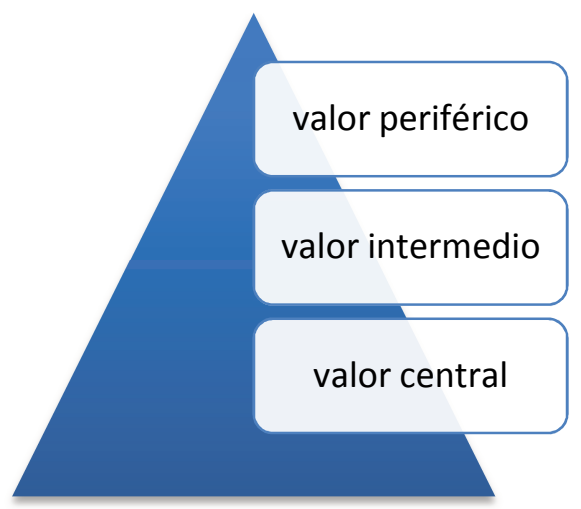

Figura 1. Jerarquización de valores en una sociedad

Naturalmente, ese gradatum organiza también el estatus de los diferentes grupos de valores. Los centrales, en tanto que nucleares a la cosmovisión de los individuos, se encuentran fuertemente arraigados en los grupos sociales. A medida que se apartan de ahí, los valores empiezan a perder capacidad definitoria y estabilidad dentro del imaginario social. La configuración de ese conjunto de valores transcribe la mentalidad de una época y una sociedad concretas (García Marcos 2015).

La metodología australiana operó con cuestionarios. Cada valor se correspondía con un ítem para el que solicitaba una puntuación. De ahí se obtenía un listado que permitía ordenar y clasificar los valores, discriminando su grado de centralidad. En comparación con los estudios de otras disciplinas sociales, Smolicz optó por un abanico considerablemente amplio de valores, lo que permitió acceder a un conocimiento más exhaustivo de la cosmovisión de los grupos sociales.

\section{METODOLOGÍA}

El presente trabajo propone una aproximación empírica al lugar que ocupa el lenguaje dentro de los valores de la comunidad de habla de Almería capital. Para ello se ha tomado una muestra de 100 sujetos, lo que supone un $0,019 \%$ respecto de 
192697 habitantes de la ciudad, con lo que está garantizada su fiabilidad estadística. La muestra la tomó durante 2013 y 2014 un equipo del CALA integrado por S. Otero, M. Mendy y yo mismo.

Esos 100 sujetos han sido estratificados atendiendo a cuatro factores sociales: edad, sexo y formación académica, habituales en las investigaciones sociolingüísticas, junto a la procedencia, dado el fuerte peso de la población inmigrada en la comunidad de Almería durante las dos últimas décadas. Los tres primeros factores fueron estratificados censalmente, siguiendo los parámetros utilizados en la sociolingüística variacionista. La procedencia comportó mayores dificultades. En 2006 García Marcos y Carmona censamos 101 etnias distintas en Almería. Evidentemente, ni podía atomizarse la muestra ni agruparla de manera indiscriminada. En García Marcos (2010) se propuso tabular esa variable atendiendo a criterios antropológicos, dentro de un trabajo más amplio de investigación interdisciplinar sobre las migraciones en el Sureste español. Esa opción resultó útil, por lo que parece conveniente adoptarla aquí. De ese modo, los factores sociales tomados en este estudio quedan subdivididas como sigue, con un $+/-5 \%$ respecto de desviación respecto del Censo de 2013 y los Cuadernos de la Fundación BBVA (2009).

Tabla 1. Estratificación de la muestra

\begin{tabular}{|l|l|l|}
\hline Sexo & Hombres & 48 \\
\hline & Mujeres & 52 \\
\hline Edad & -20 & 22 \\
\hline & $20-45$ & 39 \\
\hline & $45-65$ & 24 \\
\hline & $65-$ & 15 \\
\hline Estudios & Sin estudios & 17 \\
\hline & Primaria & 27 \\
\hline & Secundaria & 38 \\
\hline & Universidad & 13 \\
\hline Procedencia & Vernáculos & 64 \\
\hline & Resto-UE & 10 \\
\hline & Magrebíes & 14 \\
\hline & Susaharianos & 12 \\
\hline
\end{tabular}

Esos hablantes han respondido a un cuestionario que ha mantenido los ítems empleados por Otero (2014), lo que proporciona una base inmediata de contraste de los resultados que se obtengan. De esa manera, se propuso responder a la pregunta “¿qué considera Vd. que explica mejor lo que es su ciudad?”, puntuando de 1 (poco) a 5 (mucho) los siguientes valores: religión, lengua, cultura, deporte, gastronomía, historia y paisaje. 


\section{ANÁLISIS}

Una primera aproximación a la distribución general de valores en la comunidad de Almería presenta una fuerte diversidad y estratificación, tal y como queda recogido en el cuadro siguiente.

Tabla 2. Valores centrales en la comunidad de Almería. Datos generales

\begin{tabular}{|l|c|c|}
\hline \multicolumn{1}{|c|}{ Valor } & Rango & Media \\
\hline Lengua & 1 & 4,6 \\
\hline Historia & 2 & 4,23 \\
\hline Deporte & 3 & 4,34 \\
\hline Gastronomía & 4 & 3,43 \\
\hline Cultura & 5 & 2,97 \\
\hline Paisaje & 6 & 3,13 \\
\hline Religión & 7 & 2,88 \\
\hline
\end{tabular}

Como puede observarse en la tabla el valor central está claramente ubicado en torno a la lengua, dentro de un espectro que, en todo caso, se muestra distribuido alrededor de tres grandes bloques. Fijada esa posición central de la lengua, un segundo grupo estaría integrado por la historia y el deporte, con apreciaciones relativamente parejas y constantes, tendencialmente próximas a la posición central. En el extremo contrario figurarían los restantes parámetros socioculturales sometidos a examen, la cultura, la gastronomía, el paisaje y, en especial, la religión, que ocupa el último de los rangos.

Esa situación, tan amplia en lo cuantitativo, es de suponer que se encuentre extendida a través de todos los grupos sociales de la comunidad. Lo está, aunque no exenta de matizaciones, como de hecho se comprobará de inmediato al abordar los resultados arrojados por las variables sociales que ha incorporado este trabajo.

El factor sexo introduce las primeras puntualizaciones sustanciales, aportando los primeros parámetros de estratificación sociolingüística.

Tabla 3. Valores centrales en la comunidad de Almería. Factor sexo

\begin{tabular}{|c|l|l|}
\hline Rango & \multicolumn{1}{|c|}{ Hombres } & \multicolumn{1}{c|}{ Mujeres } \\
\hline 1 & Lengua & Lengua \\
\hline 2 & Deporte & Cultura \\
\hline 3 & Cultura & Historia \\
\hline 4 & Gastronomía & Religión \\
\hline 5 & Historia & Gastronomía \\
\hline 6 & Paisaje & Deporte \\
\hline 7 & Religión & Paisaje \\
\hline
\end{tabular}


En efecto, la tabla anterior parece reproducir algunos tópicos sociales especialmente asentados en el imaginario colectivo. Hombres y mujeres organizan sus valores de manera sustancialmente distinta, al menos en este primer nivel amplio de análisis. Tanto es así que suministran listados ostensiblemente diferentes.

Dejando al margen la posición central de la identidad lingüística, la ordenación de valores masculina y femenina no coincide en ningún nivel a partir del rango 2 . Mientras que las mujeres se inclinan por la cultura y la historia como preferencias a continuación de la lengua, los hombres en cambio prefieren el deporte y la historia. Llaman la atención, además, varios datos. Los hombres parecen ser más sensibles a las cuestiones culinarias, o cuando menos las valoran más. Por otra parte, en las mujeres la religión abandona el último lugar, ascendiendo hasta el rango 4, por encima de la gastronomía, el deporte o el paisaje. En los hombres, sin embargo, la religión obtiene los peores promedios de toda la muestra, como recogen los cuadros siguientes.

La edad introduce algunos matices muy significativos a todo lo expuesto hasta este momento. En esta ocasión la tabla 4 resume a continuación los datos correspondientes a su situación en la comunidad de Almería.

Tabla 4. Valores centrales en la comunidad de Almería. Factor social edad

\begin{tabular}{|c|l|l|l|l|}
\hline Rango & \multicolumn{1}{|c|}{ Hasta 20 } & \multicolumn{1}{c|}{ De 20 a 45 } & \multicolumn{1}{c|}{ De 45 a 65 } & \multicolumn{1}{c|}{ Más de 65 } \\
\hline 1 & Deporte & Deporte & Lengua & Lengua \\
\hline 2 & Lengua & Lengua & Cultura & Historia \\
\hline 3 & Paisaje & Cultura & Historia & Cultura \\
\hline 4 & Cultura & Historia & Deporte & Deporte \\
\hline 5 & Gastronomía & Paisaje & Gastronomía & Gastronomía \\
\hline 6 & Historia & Gastronomía & Paisaje & Religión \\
\hline 7 & Religión & Religión & Religión & Paisaje \\
\hline
\end{tabular}

La lengua se mantiene como gran indicio identitario dentro de la comunidad, siempre en los primeros lugares de las valoraciones de todos los grupos generacionales. Con todo, es el tercero de ellos donde predomina con mayor claridad y de manera más inequívoca.

El resto de valores se mantienen, grosso modo, siguiendo la tendencia general de la comunidad, excepto precisamente el deporte. Ahí se registra una determinante influencia generacional, que incrementa de manera más que ostensible e intensa su valoración en los dos grupos más jóvenes de sujetos, en especial entre aquellos que están por debajo de los 20 años de edad. Tanto es así que su ordenación de rangos es diferente a la de los restantes grupos generacionales, como de hecho muestra el cuadro siguiente. 
La cultura, por su parte, aporta matices muy relevantes para terminar de aquilatar la asignación de valores psicosociales en la comunidad de Almería.

Tabla 5. Valores centrales en la comunidad de Almería. Factor social cultura

\begin{tabular}{|c|l|l|l|l|}
\hline Rango & \multicolumn{1}{c|}{ Sin estudios } & \multicolumn{1}{c|}{ E. primarios } & \multicolumn{1}{c|}{ E. secundarios } & \multicolumn{1}{c|}{ E. superiores } \\
\hline 1 & Religión & Cultura & Lengua & Lengua \\
\hline 2 & Lengua & Deporte & Cultura & Historia \\
\hline 3 & Deporte & Lengua & Deporte & Cultura \\
\hline 4 & Paisaje & Historia & Historia & Deporte \\
\hline 5 & Cultura & Religión & Gastronomía & Gastronomía \\
\hline 6 & Historia & Paisaje & Paisaje & Paisaje \\
\hline 7 & Gastronomía & Gastronomía & Religión & Religión \\
\hline
\end{tabular}

El espectro cultural muestra una distribución bastante asimétrica. De un lado, hay una serie de valores más o menos estabilizados y constantes, casos del paisaje, la gastronomía o la cultura. De otro, en el extremo más activo del factor cultural, otros valores sí que aparecen claramente condicionados por su intervención.

La tendencia contraria se pone de manifiesto entre la historia y la lengua. Mantienen valores altos, como en general ha sucedido en toda la muestra. En esta ocasión, además, refuerzan su presencia a medida que suben en la pirámide cultural. Son los hablantes universitarios - o de secundaria - quienes más aprecian de manera preferente ambos componentes de la vida comunitaria.

Por último, la procedencia introduce las últimas puntualizaciones significativas a esa panorámica de estratificación de valores.

Tabla 6. Valores centrales en la comunidad de Almería. Factor social procedencia

\begin{tabular}{|c|l|l|l|l|}
\hline Rango & \multicolumn{1}{|c|}{ Españoles } & \multicolumn{1}{c|}{ Europeos } & \multicolumn{1}{c|}{ Magrebíes } & \multicolumn{1}{c|}{ Subsaharianos } \\
\hline 1 & Lengua & Lengua & Historia & Historia \\
\hline 2 & Historia & Deporte & Lengua & Cultura \\
\hline 3 & Deporte & Cultura & Cultura & Historia \\
\hline 4 & Cultura & Gastronomía & Deporte & Lengua \\
\hline 5 & Paisaje & Historia & Religión & Religión \\
\hline 6 & Gastronomía & Paisaje & Paisaje & Gastronomía \\
\hline 7 & Religión & Religión & Gastronomía & Paisaje \\
\hline
\end{tabular}

La procedencia ha sido el factor social más activo de todos los muestreados. Ello pone de manifiesto, de entrada, el fuerte condicionamiento de los parámetros culturales entre los que se desenvuelven los individuos, no solo a la hora de asignar sus valores sociales, sino probablemente también a la hora de convertirlos en conducta 
social en la vida cotidiana. En lo que aquí concierne, las cuestiones lingüísticas mantienen una posición predominante entre los europeos, y un alto rango para los magrebíes, descendiendo de manera significativa en el colectivo subsahariano.

\section{CONCLUSIONES}

Los datos de Otero (2014) coinciden con el diagnóstico general que acaba de mostrarse, sobre todo en lo concerniente a la lengua. Tanto en la capital como en el resto de la provincia aparece como el gran signo identitario de la comunidad incluso por encima de otros valores que, en principio, deberían estar muy arraigados en el imaginario colectivo, caso de la religión o la historia. Es más, esa consideración absolutamente nuclear de las cuestiones lingüísticas es la mayor constante entre ambos estudios. A partir de ahí, la contraposición con el mundo rural investigado por Otero es bastante evidente, sobre todo en algunos aspectos. En el resto de la provincia, la religión ocupaba posiciones más altas entre hablantes vernáculos, e igualmente preeminentes entre los inmigrados. De igual modo, el entorno rural propicia una mayor sensibilidad hacia el paisaje y, consecuencia de ello, su mejor ubicación en el conjunto de valores.

La completa centralidad del lenguaje puede tener varias causas. De una parte, parece ser una constante entre los hablantes hispanos, como en su día constataron Clyne y Kipp (2011), precisamente al analizar las minorías inmigradas en Australia. De otra, el contexto particular de España durante la última década ha situado en primera línea del debate social la cuestión de la lengua, lo que de inmediato se traslada al imaginario y al sistema de valores colectivos. En tercer lugar, la propia situación migratoria genera sentimientos identitarios en torno a la lengua, llegando a generar movimientos de convergencia interdialectal que no se producen en sus países de origen. En el caso de los inmigrados musulmanes ha de agregarse el alto valor religioso de su lengua, circunstancia que refuerza todo lo anterior. Por último, el descenso relativo entre los hablantes subsaharianos probablemente esté vinculado a su propia dinámica sociolingüística de origen, en la que los idiomas tienen un carácter mucho más coyuntural, dentro de estados sin identidad idiomática claramente establecida. Por supuesto que todo ello aporta datos más que determinantes para encaminar la necesaria planificación de un contacto lingüístico tan profuso como el registrado en Almería.

La propuesta de J.J. Smolicz se ha mostrado, una vez más, altamente pertinente. Es probable que los datos de Almería requieran de investigaciones complementarias. Pero, de momento, son ya un primer basamento, un punto de partida conveniente. 


\section{BIBLIOGRAFÍA}

Alias García, A. (2016). El deporte en el conjunto de valores de una sociedad. In Actas del IV Congreso Internacional de Deporte inclusivo (pp. 132-151). Almería: Universidad de Almería.

Clyne, M. y Kipp, S. (2011). Pluricentric Languages in an immigrant context. Berlín: De Gruyter.

García Marcos, F. (1999). Fundamentos críticos de sociolingüística. Almería: Universidad de Almería.

García Marcos, F. (2010). Contacto lingüistico en el Sureste Español. Almería: CEMyRI.

García Marcos, F. (2014). Sociolingüistica. Madrid: Síntesis.

García Marcos, F. y Carmona, J.P. (2002). Contacto de lenguas y tipología lingüística en Almería. In L. Durán, J. de Dios Pamies Bertrán y A. Manjón Pozas (eds.), Nuevas tendencias en la investigación lingüistica (pp. 67-79). Granada: Método.

Mendy, M. (2014). Core value entre la población inmigrada de Neuchâtel (Suiza). Trabajo de grado inédito. Universidad de Almería.

Otero, S. (2014). Las investigaciones de core value aplicadas al aula multilingüe y multicultural. Trabajo de maestría inédito. Universidad de Almería.

Smolicz, J.J. (1979). Culture and Education in Plural Society. Culture and Curriculum Series. Canberra: Curriculum Development Centre. 